\title{
Fuzzy multiple criteria decision making approach in environmental risk assessment
}

\author{
Andrejs Radionovs, Oleg Uzhga-Rebrov \\ Rezekne Higher Education Institution, Faculty of Engineering. \\ Address: Atbrīvošanas aleja 115/K-3, Rezekne, LV-4601, Latvia
}

\begin{abstract}
Being able to evaluate risks is an important task in many areas of human activity: economics, ecology, etc. In case of a sufficient amount of source information the risk is evaluated using statistical methods. However, in reality the sufficiency of statistical data in risk assessment is more exceptional than normal. In such cases experts' assessment make the only source of data. Experts are able to provide the necessary for analysis data due to their professional knowledge and experience. Certain amount of factors, which is to be evaluated by an expert (experts), significantly affects the process of experts' assessment. If a big number of relevant factors occur, an expert may face a problem of defining links between "factors" and "outcome". Fuzzy multiple criteria decision making approach can be used to solve the problem. Ecological risk assessment towards human health in case of gaseous substances escape at a chemical factory using hierarchical method and fuzzy multiple criteria decision making approach has been analyzed in the article.
\end{abstract}

Keywords: Fuzzy logic, risk assessment, fuzzy representation of knowledge.

\section{INTRODUCTION}

Due to the increasing both of human industrial activity and factors promoting the environment pollution, a necessity of risk assessment becomes more essential. There exist quite a big amount of investigations devoted to the analysis of ecological risks, for example [1] and [2]. In order to make the ecological risks analysis complete, a lot of statistical data are needed. There are many papers describing the ecological risks analysis through the assessment of statistical data, for example [3]. Appraisal of a building and its insurance could be an example of statistical method. For instance, there is enough information available all over the world on the possibility of fires in various types of buildings and their market values. Estimated losses caused by fire can be easily calculated as average losses related to previous cases. Due to the rates obtained the risk can be easily evaluated and the cost of the insurance can be determined. Unfortunately, when speaking about the analysis of ecological risks the enough availability of statistical data is more exceptional than normal. Uniqueness of many situations and their rare occurrence does not allow using an effective statistical instrument for getting certain assessments. In order to cope with the lack of objective information, experts' judgments are often used. On the basis of their professional knowledge and experience, experts are able to provide the necessary for analysis data. Quite a big number of methods are meant for acquiring and using ambiguous probabilistic assessments, including interval probability, probability of second degree, etc. Difficulty of these methods and a bad interpretation of uncertain results make the methods imperfect. In 1965, L.Zadeh [4] proposed a principally new conceptual basis for dealing with imprecise information - fuzzy set theory. This theory was widely developed during the past years. Nowadays, fuzziness is used practically in all fields of scientific and practical activity, including risk assessment. The method contains many advantages, for example: includes qualitative variables in the risk assessment; works with fuzzy information; operates with linguistic variables; allows fast modeling of complex dynamic systems; however, there exist disadvantages, too: it is reasonable to use the method purely with a small amount of the risk influencing factors. Increasing of number of the mentioned factors leads to a complicated process of setting the rules, and therefore makes the possibility to make a mistake more common, and an expert may face a problem of determining the links "factors - final result". So, what should we do if it is necessary to evaluate risk with a big number of factors? In such case hierarchical fuzzy multi-criteria decision making approach may be used.

There exists quite a big amount of papers describing the usage of this method in various fields, e.g. For example, paper [5] deals with using the method to diagnose the disease aphasia.

Hierarchical fuzzy multi-criteria decision making approach in the risk assessment towards human health in case of the gas substances escape at a chemical 
factory has been used in the present article for the ecological risks assessment.

\section{FUZZY LOGIC AND FUZZY SETS}

Nowadays there are a lot of textbooks and reference books available on the theory of fuzzy sets and fuzzy logic. Work [6] can be used as an example, but a more detailed and complete description of the theory can be found in [7]. Fuzzy sets theory represents a number of mathematical principles for knowledge representation based on degrees of a membership. The definition "fuzzy logic" has appeared in the sixties with the 1965 proposal of fuzzy set theory by Lotfi A. Zadeh, when he published his work "Fuzzy Sets" in order to provide a model for inexact and not precise concepts and subjective judgments.

Fuzzy Sets deals with degrees of membership to certain class and degrees of truth. Fuzzy logic uses fuzzy rules that should receive desire results from input linguistic data or variables.

The linguistic variable is a variable, which values sentences in a natural language. For example, linguistic variable "Age" contains values: young, old, etc.

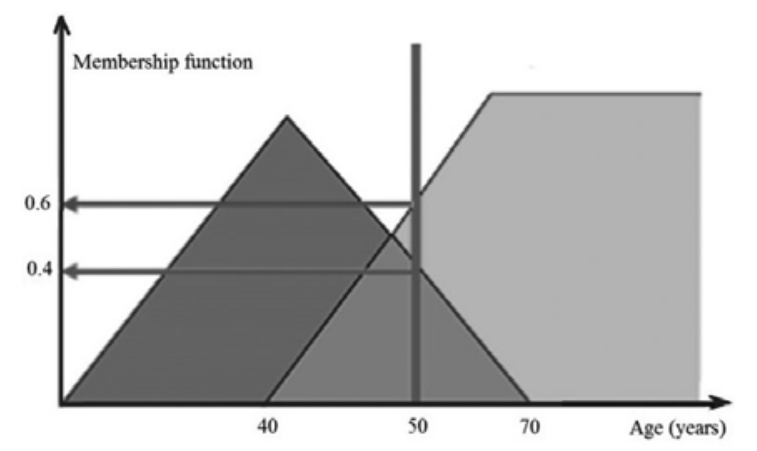

Fig. 1. Fuzzy age-classification scheme

The advantage of fuzzy methods is their reflection in human mind and its ability to store and process information that is uncertain, imprecise, and resistant to classification [8]. Fuzzy logic is an alternative to the Boolean logic where every proposition must be either true or false. However, fuzzy logic implies that things can be at the same time "true" and "false" with a certain membership degree [9]. For example, in Boolean logic a 50 year-old person can be young or old depending on the scale. But in fuzzy logic (as it is shown in Fig. 1) a man can be simultaneously old with degree of membership of 0.6 and young with 0.4.

Fuzzy set theory provides a way to use imprecise and uncertain information generated by the system and human judgments in a precise way. When the available environmental data availability do not provide an appropriate statistical information, fuzzy approaches can solve this problem, since it works well for addressing poorly defined parameters and linguistic variables [10].

One of the advantages of Fuzzy logic is its ability to work with different kinds of parameters (e.g. environmental, health), quantitative, qualitative and crisp values.

\section{MULTIPLE CRITERIA DECISION MAKING APPROACH}

Many factors influence the risk assessment of human health in case of the gas substances escape at a chemical factory. Factors are represented by Fig. 2 . Experts have to determine $3^{12}=531441$ rules by using a one-level fuzzy risk assessment methodology with 12 input variables and 3 definite for each variable fuzzy sets. This phenomenon was called by Bellman [11] the "curse of dimensionality". The rule base rapidly overloads the memory and makes the fuzzy system unusable. The problem can be solved if the 12variable fuzzy system is reduced into a hierarchical fuzzy system. For example, 8 hierarchically connected low-dimensional fuzzy systems as shown in Fig. 3, the number of fuzzy rules reduces to $3 *\left(3^{3}\right)+5^{*}\left(3^{2}\right)=126$ rules in total. This result is gained by dividing a big system into components, and by using the mechanism of fuzzy process towards each system component and level.

Further an example of fuzzy methodology towards one of the system's components is shown by a dotted line at Fig.3. 


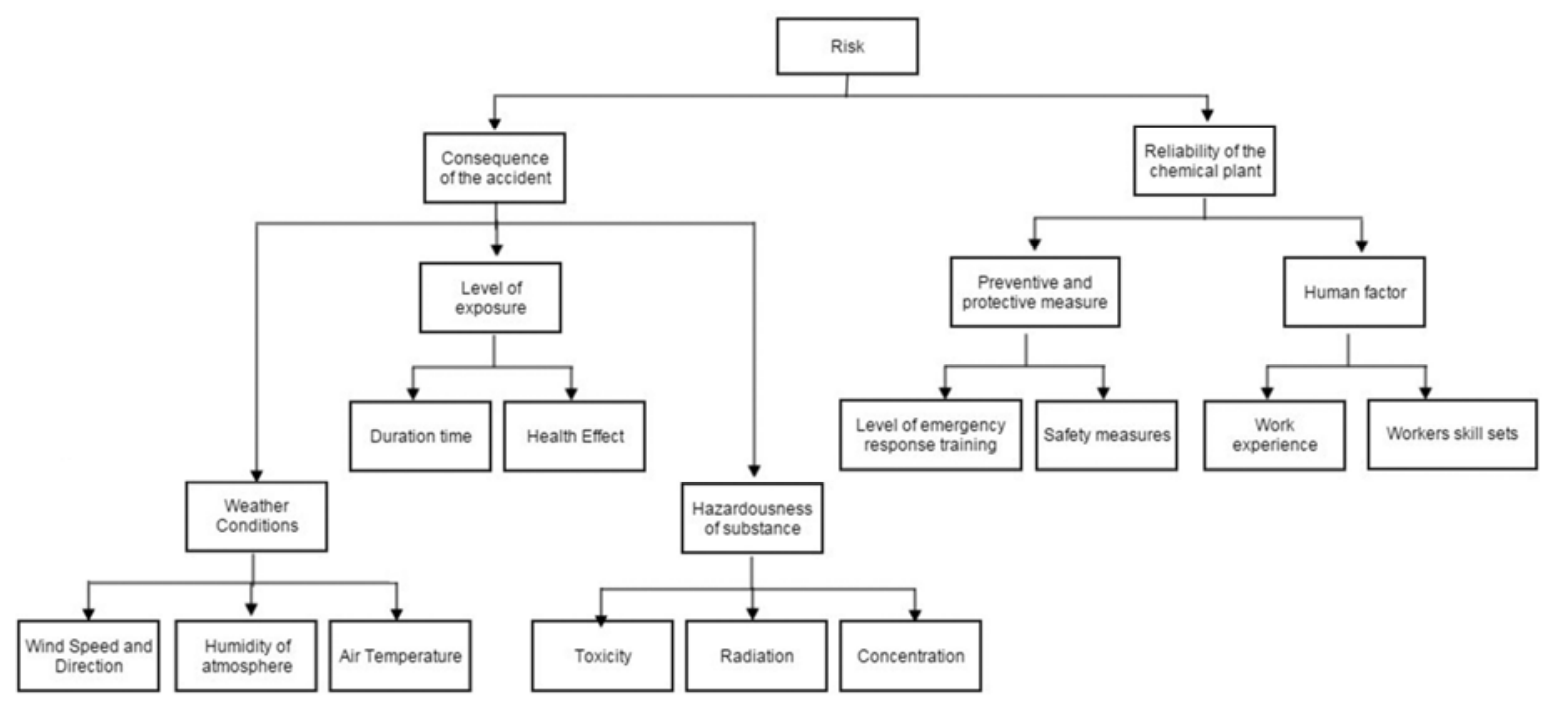

Fig. 2. Risk assessment scheme for escape of gas substances in chemical plants

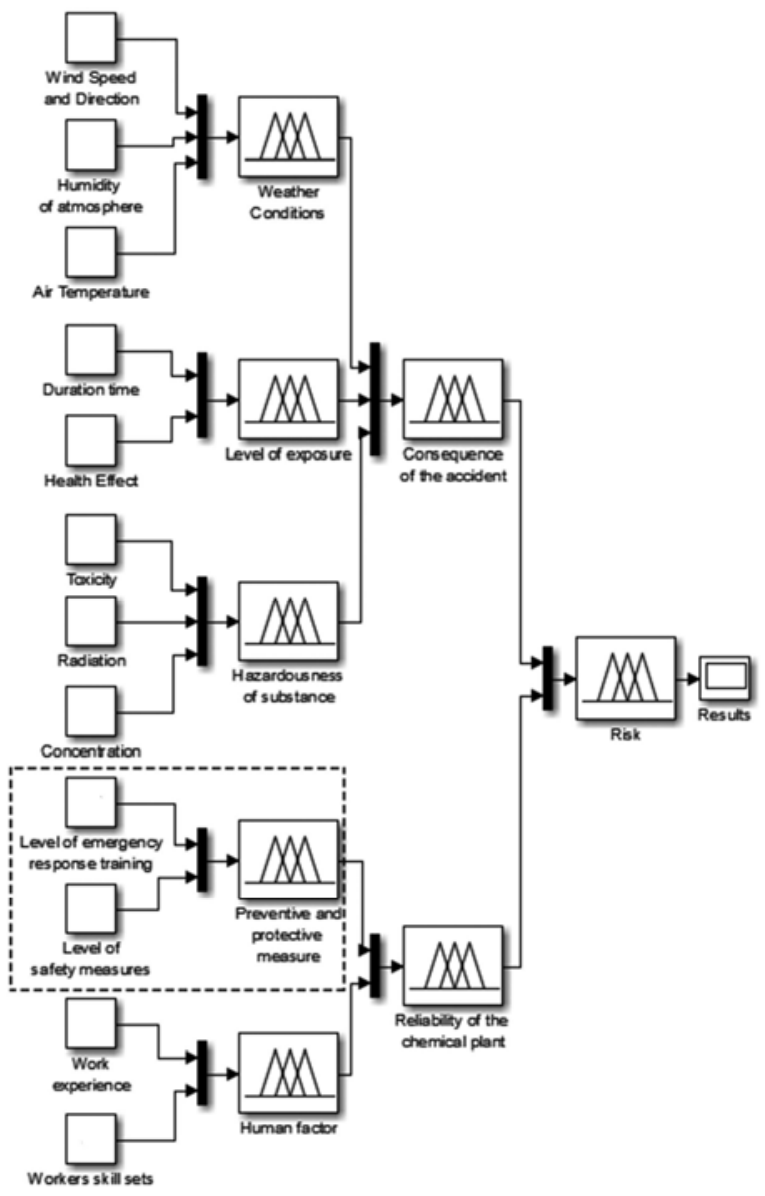

Fig. 3. Hierarchical Fuzzy Rule-Based Model Architecture

\section{FUZZY PROCESS FOR ONE SYSTEM'S COMPONENT}

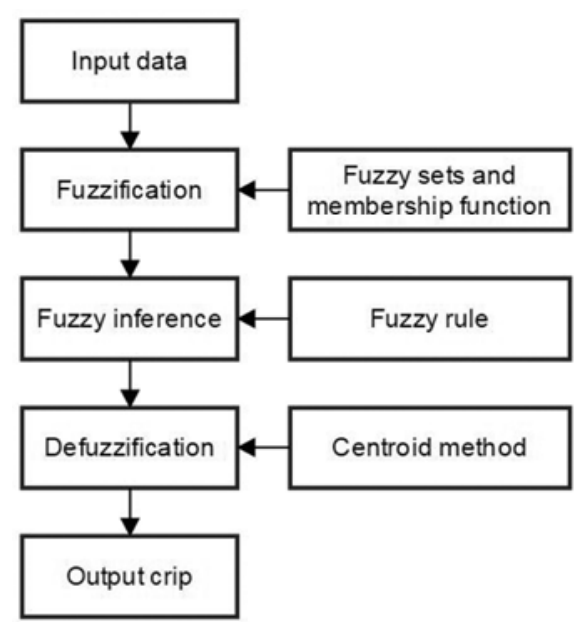

Fig. 4. Representation of fuzzy methodology

Projecting of uncertain system mainly includes two operations: knowledge base derivation and the selection of fuzzy inference process to perform fuzzy reasoning [12].

Successful projecting of uncertain system for certain Application Domain is a complicated process of several levels where the designer faces a big amount of alternative strategies of realization [13].

Fig. 4 shows that the proposed uncertain model includes various levels. Parameters of input and output should be determined at the first step, and then transformed from original values into linguistic categories by creating fuzzy sets for each of them. This process is called fuzzification. Secondly, it is necessary to determine the rules. These rules will 
allow system to "reason" or going from the input variables to the output variable. For this purpose Mamdani's fuzzy inference method will be used. For the final step, the obtained value must be defuzzificated or converted back to crisp value in order to be used in the next level of fuzzy process as shown in Fig. 3.

All the steps have been implemented by using fuzzy toolbar available in Matlab and described further.

\section{A. Definition of Input}

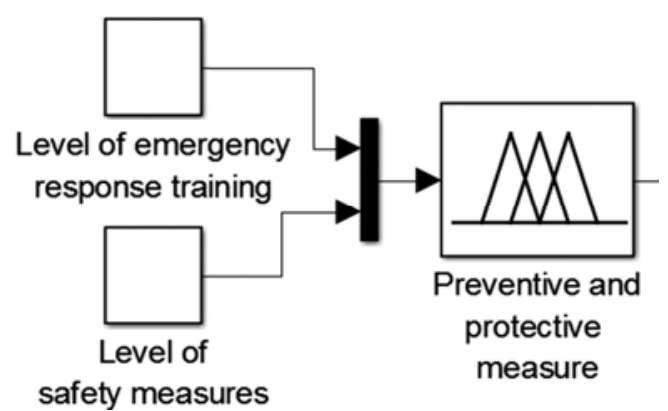

Fig. 5. Part of risk assessment scheme

At the first step the input and output variables (shown in Fig. 5) must be defined and then converted from the original values in to the linguistic categories by creating fuzzy sets for each of them. As it is shown in Tables I and II, fuzzy sets for Level of emergency response training and Level of safety measures inputs variables were made.

TABLE I

CRITERIA OF EMERGENCY RESPONSE TRAINING LEVEL

\begin{tabular}{|c|l|}
\hline $\begin{array}{c}\text { Emergency response } \\
\text { training levels (E) }\end{array}$ & \multicolumn{1}{|c|}{ Criterion } \\
\hline $\begin{array}{c}\text { First Responder } \\
\text { Awareness }\end{array}$ & $\begin{array}{l}\text { Personnel likely to discover a release } \\
\text { and call for assistance. They would } \\
\text { leave the area, call for help, and keep } \\
\text { others to get out of the area. Min.2-4 } \\
\text { hours training }\end{array}$ \\
\hline II & $\begin{array}{l}\text { Personnel are to respond in a defensive } \\
\text { manner without actually trying to stop } \\
\text { the release. Their primary function is to } \\
\text { Operations }\end{array}$ \\
$\begin{array}{c}\text { contain the release from a safe distance, } \\
\text { keep it from spreading, and prevent } \\
\text { exposures. May be involved in } \\
\text { decontamination. Min. 8-hours training }\end{array}$ \\
\hline III & $\begin{array}{l}\text { Their main purpose is rescue or to stop } \\
\text { the release. They will approach to stop } \\
\text { the release by plugging, patching or } \\
\text { shutting down the process. Min. 24- } \\
\text { hours training }\end{array}$ \\
\hline
\end{tabular}

TABLE II

Criteria of SAFETy MEASURES LEVELS

\begin{tabular}{|c|l|}
\hline $\begin{array}{c}\text { Safety measures } \\
\text { levels of the } \\
\text { chemical plant }(\mathrm{S})\end{array}$ & \multicolumn{1}{|c|}{ Criterion } \\
\hline High & $\begin{array}{l}\text { The plant fully corresponds to the safety } \\
\text { requirements; }\end{array}$ \\
\hline
\end{tabular}

\begin{tabular}{|c|l|}
\hline Medium & $\begin{array}{l}\text { Generally, the plant corresponds to } \\
\text { necessary safety conditions; }\end{array}$ \\
\hline Low & $\begin{array}{l}\text { The plant does not correspond to } \\
\text { necessary safety requirements; }\end{array}$ \\
\hline
\end{tabular}

\section{B. Output Definition}

Parameter Preventive and protective measure represents the result of hierarchical analysis at the present level (shown by Figure 5). As it is shown in Table III, for output fuzzy sets were created.

TABLE III

Criteria Preventive and Protective Measure LeVel

\begin{tabular}{|c|l|}
\hline $\begin{array}{c}\text { Preventive and } \\
\text { protective measure } \\
\text { level (P) }\end{array}$ & \multicolumn{1}{|c|}{ Criterion of evaluation } \\
\hline H-High & $\begin{array}{l}\text { Gas escape: highly trained personnel, } \\
\text { modern safety techniques are used. }\end{array}$ \\
\hline M-Medium & $\begin{array}{l}\text { Medium level of personnel training in } \\
\text { force-majeure circumstances, } \\
\text { insufficient safety measures }\end{array}$ \\
\hline L-Low & $\begin{array}{l}\text { Weak personnel training in case of } \\
\text { chemical escape, no safety measures } \\
\text { implemented. }\end{array}$ \\
\hline
\end{tabular}

\section{Fuzzification}

In the fuzzification process, the membership functions defined on the input, and output variables are applied to actual values to determine the degree of truth for each rule. Gaussian type of membership function has been used in the present paper. Fig. 6 presents the fuzzy sets and its membership function for one of input variables (chemical plant safety measures) used in this part of fuzzy risk assessment.

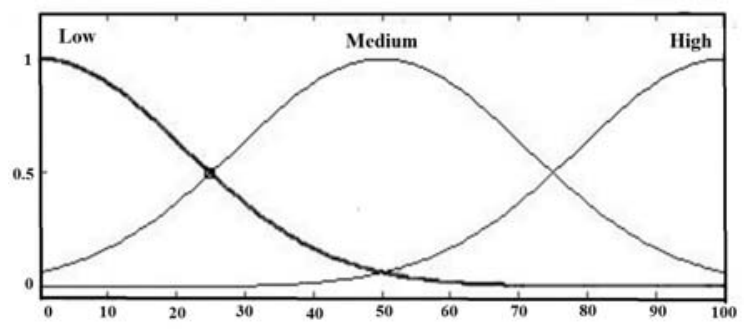

Fig. 6. Graphs of membership functions of chemical plant safety measures levels

\section{Setting Up the Rules}

Fuzzy logic is a decisional system based on linguistic rules [14]. Therefore, next step is to connect input and output fuzzy sets by rules. One type of fuzzy inference method is called the Mamdani's inference method or max-min inference method. This method is the most commonly seen fuzzy methodology. It was proposed in 1975 by E. Mamdani [15] as an attempt to control a steam engine and boiler combination by synthesizing a set of linguistic control rules obtained from experienced human operators.

The mostly used rule format is: "IF $x$ and $y$ THEN $z$ ", where $x$ and $y$ is the premise and $z$ is the 
consequence. For example: "IF emergency response training levels is III AND Safety measures level is High THEN Preventive and protective measure level is High.

All the rules can be seen in Table IV.

TABLE IV

A SYSTEM OF RULES OF FUZZY INFERENCE

\begin{tabular}{|c|c|c|c|}
\hline \multirow{2}{*}{$\begin{array}{c}\text { Emergency } \\
\text { response training } \\
\text { level (E) }\end{array}$} & \multicolumn{3}{|c|}{ Safety measures levels (S) } \\
\cline { 2 - 4 } & High & Medium & Low \\
\hline I & $\mathrm{M}$ & $\mathrm{L}$ & $\mathrm{L}$ \\
\hline $\mathrm{II}$ & $\mathrm{H}$ & $\mathrm{M}$ & $\mathrm{L}$ \\
\hline $\mathrm{III}$ & $\mathrm{H}$ & $\mathrm{H}$ & $\mathrm{M}$ \\
\hline
\end{tabular}

\section{E. Defuzzification}

The defuzzification process is the conversion of the fuzzy output set to a crisp number. Defuzzification process and can be done by using different methods. Some of them are: max, mean-max membership principle, centroid method, medium, weighted average method, center of the largest area method and other methods [16].

In this paper a centroid method has been selected as the most common and widely used method of defuzzification. According to the centroid method the crisp value of the output variable is computed by finding the variable value of the center of gravity of the membership function for the fuzzy value. More complete description of this algorithm is available in [17].

The defuzzification process results are represented at the top of Fig. 7. For example, if parameter Emergency response training level makes 75 units and parameter Safety measures level - 63, then Preventive and protective measure level will be 56.5 units.
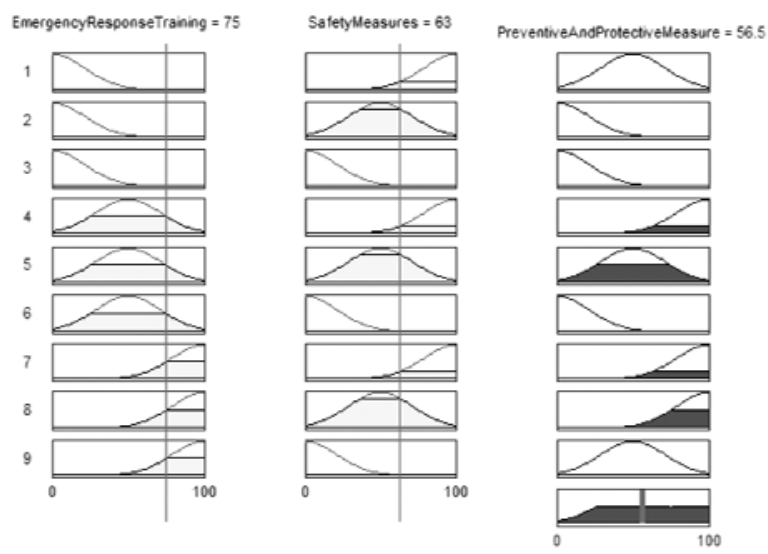

Fig. 7. Application of centroid method to the input variables

\section{RISK LEVEL}

Considering the described before Fuzzy Process at each unit of the system illustrated by Fig. 3, we get a final result, which in the present paper represents the risk level.
The risk level is represented as a surface as it is shown in Fig. 8. as a three-dimensional representation of the risk, consequence of the accident and reliability of the chemical plant.

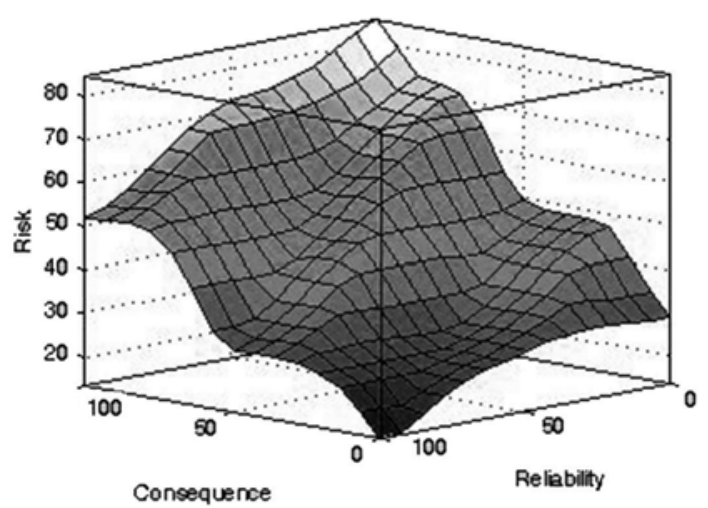

Fig. 8. Three-dimensional representation of the risk, consequence of the accident and reliability of the chemical plant

\section{CONCLUSION}

The application of hierarchical fuzzy multi-criteria decision making approach to the assessment of risk of environment and people's health it in case of potential accident and escape of gaseous substance allows to significantly facilitate the process of risk assessment when a big amount of source data are available, to represent the results in a simple and understandable way, which is appropriate for determining the needed activities for certain results of analysis.

One of the main advantages of using linguistic variables in hierarchical form is that such expressions are more intuitive that makes it easier for experts to give their evaluations in unclear and complex situations, in which numerical estimations or crisp estimations are hard to obtain. Also using the hierarchical method a number or rules that experts must define is considerably reduced. In this work rules are reduced from 531441 to 126 rules.

This methodology can be used not only by public authorities but also by plant managers, since it is a method that allows evaluating the risk level of the site and enables to understand whether the safety measurements are suitable. This method can be used as a preliminary risk assessment tool to expose situations where more complete analysis is needed. Also this methodology can be used in the case when a thoughtful decision of a risk level reduction is needed.

\section{REFERENCES}

[1] Carlon, C., Critto, A., Marcomini, A., Nathanail, P. "Risk based characterization of contaminated industrial site using multivariate and geostatistical tools", Environmental Pollution, vol. 111 (3), 2001, pp. 417-427.

[2] Burningham, K., Thrush, D. "Pollution concerns in context: A comparison of local perceptions of the risks associated with living close to a road and a chemical factory", Journal of Risk Research, vol. 7 (2), 2004, pp. 213-232. 
[3] Martin B., Pearson A., Bauer B. "An Ecological Risk Assessment of Wind Energy", The Nature Conservancy Helena, Montana, pp.146, 2009

[4] L. Zadeh, "Fuzzy Sets" Information Control, 1965, 8, pp. 338 -353 .

[5] M.R. Akbarzadeh-T, M. Moshtagh-Khorasani "A hierarchical fuzzy rule-based approach to aphasia diagnosis.", Journal of Biomedical Informatics, Vol. 40, No. 5, 2007, pp. 465-475

[6] M. Ganesh, Introduction to Fuzzy Sets and Fuzzy Logic. PHI Learning Pvt. Ltd, 2006, 256 p.

[7] G.J. Klir, Y. Bo, Fuzzy Sets and Fuzzy Logic. Prentice Hall, $1995,592 \mathrm{p}$.

[8] T.E. McKone, A.W. Deshpande,, 2005. Environ. Sci. Technol. 39 (2) $42 \mathrm{~A}$

[9] L.A. Zadeh, 1983. The Role of Fuzzy Logic in the Management of Uncertainty in Expert Systems, Fuzzy Sets and Systems 11, 199.

[10] L. Zadeh, "Fuzzy logic and approximate reasoning" Synthese, Vol 30, 1975, pp. $407-428$.

[11] Bellman, R., Adaptive Control Processes, Princeton University Press, Princeton, 1966.
[12] Cordón O., Herrera, F., Peregrín A., "Looking for the Best Defuzzification Method Features for each Implication Operator to Design Accurate Fuzzy Models", Technical Report, DECSAI, 1999.

[13] Garibaldi, J. M. and Ifeachor, E.C., "Application of simulated Annealing Fuzzy Model Tuning to UmbilicalCord Acid-base Interpretation", IEEE Transactions on Fuzzy Systems , Vol.7, No.1. 1999.

[14] R. E. Bellman, L. A. Zadeh, "Decision making in a fuzzy environment, „Management Science, vol. 17, no. 4, 1970, pp. B141-B164

[15] Mamdani, E.H. and S. Assilian, "An experiment in linguistic synthesis with a fuzzy logic controller," International Journal of Man-Machine Studies, Vol. 7, No. 1, 1975, pp. 1-13.

[16] Klir, G. J., and Yuan, B., Fuzzy Sets, Fuzzy Logic and Fuzzy System, World Scientific Singapore, 1996.

[17] Ross, T. J., "Properties of membership functions, fuzzification, and defuzzification" in Fuzzy Logic with Engineering Applications, Third Edition, John Wiley \& Sons, Ltd, Chichester, United Kingdom, 2010, pp. 89-116. 